\title{
Trunk and hip muscles activation patterns in subjects with and without chronic low back pain: a systematic review
}

\author{
DOI: https://doi.org/10.5114/pq.2020.100280
}

\author{
Eman Kamel, Salwa Abdelmajeed, Hamed El Khozamy, Karima Hassan \\ Department of Physical Therapy for Musculoskeletal Disorders and Their Surgeries, Faculty of Physical Therapy, \\ Cairo University, Giza, Egypt
}

\section{Abstract}

The purpose of this study was to determine normal and abnormal patterns of activation of gluteus maximus (GM), hamstring (HAM), contralateral erector spinae (CES), and ipsilateral erector spinae (IES) muscles during a prone hip extension test in healthy or asymptomatic subjects and those with non-specific chronic low back pain through a systematic review. Studies were recognized by searching electronic databases (Embase, MEDLINE/PubMed, Cochrane Library, PEDro [Physiotherapy Evidence Database], and CINAHL) and scanning articles reference lists from the beginning until July 2018. Limits involved studies in the English language and performed among humans. Of 2112 citations and reference lists scanned, 15 articles were determined to be relevant to this review. From these studies, 4 investigated 157 subjects ( 88 asymptomatic and 69 with low back pain), and 11 investigated 257 healthy subjects. The results of the moderate and weak quality studies indicate that the HAM and ES muscles are activated early and almost simultaneously, but GM is consistently delayed in relation to leg movement and the other 3 muscles in healthy individuals. In low back pain subjects, CES are delayed and GM is significantly delayed in individuals who showed abnormal lumbar motions when compared with healthy ones.

Key words: activation patterns, low back pain, systematic review

\section{Introduction}

Chronic low back pain (CLBP) is the most common and exorbitant medical issue [1]. Reasons for CLBP give an impression of being intricate and multifactorial [2]. The primary causes of mechanical LBP were physical impairments, including postural abnormalities [3, 4], disturbance of motor control [5], and muscles imbalance [6].

Functional movement is never isolated because it is produced by several muscles acting as prime movers, synergists, or stabilizers that coordinate to produce an activation pattern [7]. In addition, functional strength does not require maximal activation; muscle onset and timing are more important [7]. The pattern of activation includes the timing (i.e., which muscle is activated first, second, third, etc.) and amount of muscle activity [8]. Adequate muscle activation patterns are recognized as important for the effective functioning of the lumbar spine when the synergic muscles are activated in a suitable temporal order [9].

Increased or decreased muscle activity and delayed muscular activation can change the normal movement pattern $[10,11]$. It has been noted that patients with chronic or recurrent LBP have altered patterns of extensor muscles $[8,12,13]$ and postural dysfunction $[14,15]$.

There are relatively few clinical tests that are used in practice to assess a patient's motor control. The prone hip extension (PHE) test is one of the most common tests used in LBP; it includes having a patient lying prone and lifting each leg alternatively while clinicians should look for the 'normal' activation sequence starting with ipsilateral gluteus maximus (GM), followed by ipsilateral hamstring (HAM) and contralateral erector spinae (CES), up to ipsilateral erector spinae
(IES) muscles [10]. Recent evidence suggests that the 'normal' order of muscle activation is incorrect and being debated; it has been demonstrated that there is not a consistent order of muscle recruitment during PHE in healthy individuals [13, 16-18].

The lack of a clear understanding of activation patterns used in asymptomatic subjects and those with CLBP could be a possible reason for the less successful outcomes of longterm complaints in patients with CLBP. Up to the authors' knowledge, 1 review of literature investigated the activation patterns during a PHE test but there was no quality assessment of the included studies and the author could not reach a comprehensive conclusion regarding the pattern [19].

To address changes in the lumbopelvic muscle activation patterns in LBP subjects as a part of a rehabilitation program, the nature of these changes needs to be studied. Therefore, the objective of this study was to systematically review the observational studies that investigated the onset times and pattern of activation of GM, HAM, and ES by surface electromyography during a PHE test in healthy or asymptomatic subjects and those with non-specific CLBP.

\section{Subjects and methods}

This review followed the guidelines of Preferred Reporting Items for Systematic Reviews and Meta-Analyses (PRISMA) [20].

The research considered all studies that investigated the activation pattern of GM, HAM (semitendinosus [ST] or biceps femoris [BF]), or ES muscles in healthy or asymptomatic and CLBP (male and female) subjects during a PHE test. Observational studies, studies on PHE with different positions, pa-

Correspondence address: Eman Abdallah Kamel, Department of Physical Therapy for Musculoskeletal Disorders and Their Surgeries, Faculty of Physical Therapy, Cairo University, 1 Gamaa Street, Giza, Postal Code: 12613, Egypt, e-mail: romaandrofa2020@gmail.com 
pers in the English language, and published papers were included. This review excluded studies that involved subjects with LBP due to fracture, tumour, neurological dysfunction, or sports injury; studies that used surface electromyography measurements in acute LBP; studies that applied PHE as a rehabilitation exercise; and conference proceedings.

Primary outcomes of interest were back and hip GM, HAM, ES muscles onset times measured by surface electromyography during a PHE test and the patterns of muscle activation. Secondary outcomes included back pain (severity, frequency, duration, and number of previous attacks).

\section{Information sources and search strategy}

The electronic search was conducted for the following databases: Embase, MEDLINE/PubMed, Cochrane Library, PEDro [Physiotherapy Evidence Database], and CINAHL, from inception until July 2018. The search strategy used search terms adapted to each database by a combination of Medical Subject Headings (MeSH) terms.

The search strategy for MEDLINE/PubMed, these terms were used:

(1): Activation Patterns OR Recruitment Pattern OR Movement Patterns OR Firing Order OR Electromyographic Activity,

(2): (1) AND Chronic Low Back Pain OR Non-Specific Low Backache OR Mechanical Back Pain,

(3): Prone Hip Extension Test OR Position,

(4): (1) AND Prone Hip Extension,

(5): (3) AND (Chronic Low Back Pain OR Non-Specific Low Back Pain OR Mechanical Back Pain),

(6): AND (“1976/01/01”[PDat]: “2018/07/31”[PDat]),

(7): Clinical Trial,

(8): Observational Study,

(9): Randomized Controlled Trial,

(10): Review,

(11): Systematic Review,

(12): English Abstract,

(13): Human Studies.

All papers were imported to Mendeley Desktop (version 1.17.11) and screened for duplicates. In accordance with the predetermined eligibility criteria, 2 reviewers (EK, KA) independently screened the title and abstract of each paper. Discrepancies between the reviewers were resolved by discussion. Full-text papers that met the inclusion criteria were retrieved by the same reviewers. The reference lists of the included papers were hand-searched.

Data extraction was performed independently by 2 reviewers (HK, EK) with a predesigned data collection form. The following data items were extracted: authors, journal and year, study design, sample sizes, participants' characteristics (age, sex, body mass index, weight, and height), muscles investigated, electrode position, onset time calculations, outcomes, and results. The researchers were contacted in order to obtain data where required.

A modified Effective Public Health Practice Project (EPHPP) quality assessment tool was used to evaluate the quality of the included papers [21]. EPHPP was designed specifically for observational studies and consists of 8 items: selection bias, study design and randomization process in participants selection or assessment, control of confounders, blinding of participants and outcome assessors, data collection methods, sample size, withdrawal and dropouts, and the analysis of outcome variables. On the basis of the adjusted EPHPP, each item was rated strong, moderate, or weak. Then, each paper was given a global rating. The global rating of a study was dependent on the number of weak items, whereas strong meant no weak items, moderate meant 1 or 2 weak items but not including confounders, and weak meant 3 or more weak items. Two independent reviewers (KA, EK) assessed the methodological quality of the included studies and, if there was any disagreement, a third reviewer was consulted.

\section{Data synthesis}

Descriptive data synthesis was based on the extracted data and the quality assessment of the included studies. Different activation patterns were identified in the included studies in healthy or asymptomatic and LBP subjects.

\section{Ethical approval}

The conducted research is not related to either human or animal use.

\section{Results}

\section{Study selection}

The comprehensive search identified 2871 citations, with 2112 citations evaluated after removal of duplicates. The full text of 27 articles were retrieved and evaluated for eligibility, 11 studies fulfilled the inclusion criteria. Four additional papers were identified by hand searching of the included papers reference lists. A total of 15 studies met the selection criteria (Figure 1).

\section{Study characteristics}

\section{Studies in healthy participants}

Eleven cross-sectional studies out of the 15 studies examined the pattern of trunk and hip muscles in healthy participants. In these 11 studies, 257 healthy subjects were investigated; the sample sizes varied between 14 [13] to 40 [22] subjects. The range of age was 20-36 years. In 7 studies, both genders were included [13, 16, 18, 22-25], while in 4 studies, only male subjects participated [17, 26-28]. The characteristics of the included studies are presented in Table 1.

\section{Studies comparing asymptomatic with LBP subjects}

Four cross-sectional studies out of the 15 determined studies aimed to compare the activation pattern of the IES, CES, GM, and HAM (BF or ST) muscles during PHE between asymptomatic and CLBP subjects [29-32]. In these studies, 157 subjects were investigated (88 asymptomatic and 69 LBP) aged 20-43 years. In 3 studies, both genders were included [29-31], and female gender only participated in the remaining one [32]. The characteristics of the included studies are presented in Table 2.

\section{Methodological quality}

In accordance with the criteria used to assess the methodological quality of the studies, 3 studies out of the 15 were of moderate quality [26, 27, 31], and 12 were of weak quality [13, 16-18, 22-25, 28-30, 32]; no studies were of strong quality. The results of the quality assessment are presented in Table 3. 


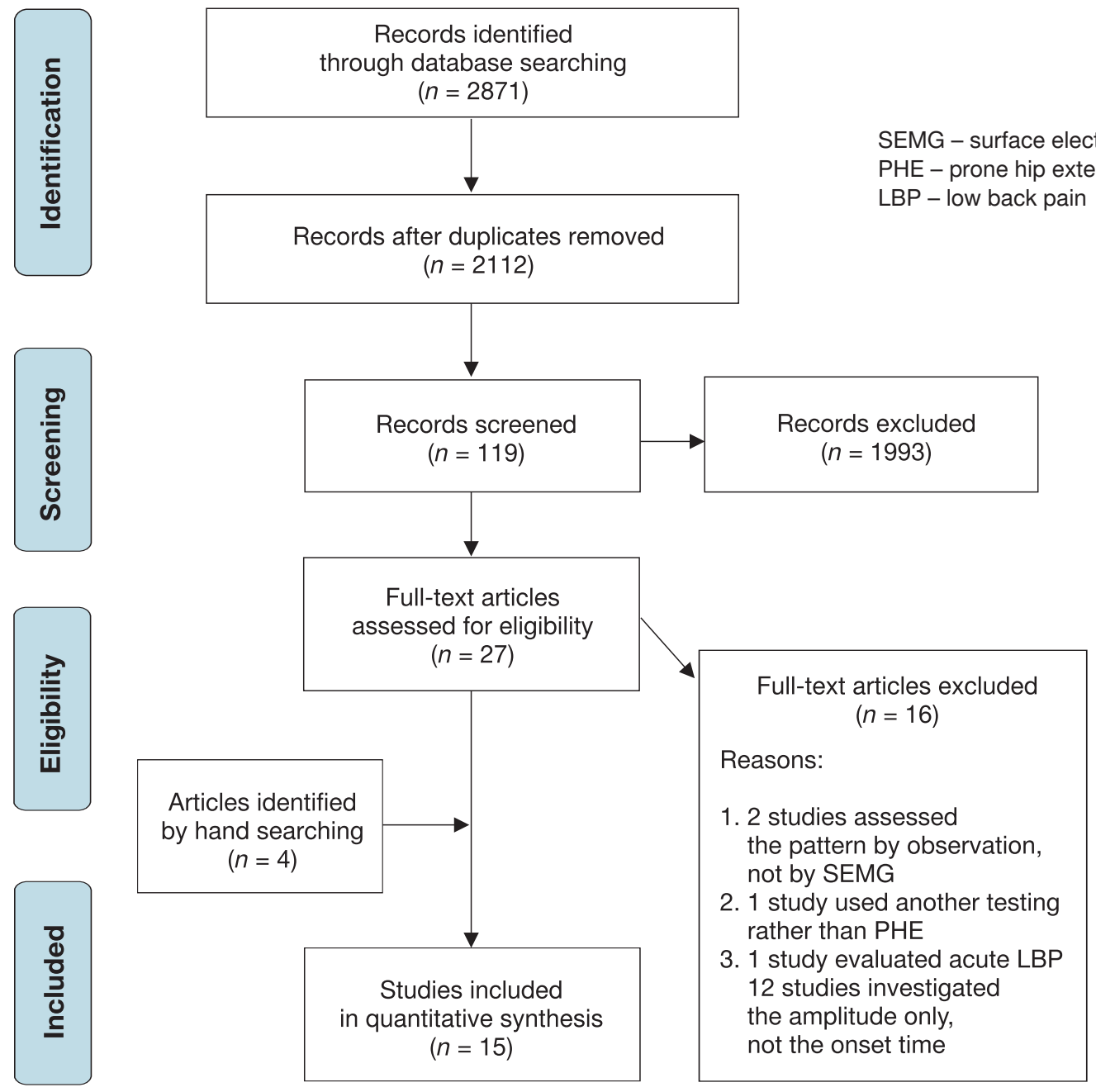

Figure 1. PRISMA flow diagram of the selection process

\section{Synthesis of results}

The results were organized into 2 categories: (a) activation patterns in healthy subjects, (b) differences in activation patterns between asymptomatic and LBP subjects.

\section{Activation patterns in healthy subjects}

Two of the studies in healthy subjects were of moderate rating quality $[26,27]$. Activation patterns during $\mathrm{PHE}$ in subjects who demonstrated specific 'abnormal' lumbar spine motion patterns (positive group) and those who did not (negative group) were investigated and the authors found that patterns used by both groups were variable [26]. There were 6 activation orders most prevalent in the positive and negative groups; these accounted for the majority of each group's total repetitions (Table 1).

The HAM, CES, and IES activated almost simultaneously in a seemingly random order, followed by GM after a delay. GM was consistently delayed relative to the other 3 muscles in the 2 groups, with the magnitude of the delay being significantly greater in the positive group compared with the negative group [26].

The effect of 3 levels of pelvis compression $(0 \mathrm{~N}, 50 \mathrm{~N}$, $100 \mathrm{~N}$ ) on the muscle firing pattern during the PHE test was investigated. There was a consistent and significant delay $(\alpha=0.05)$ in the GM muscle onset in relation with the ST muscle [27]. No significant differences were observed in the onset time of the ES muscles and ST: they activated almost simultaneously, whereas in 13 out of 20 subjects, IES contracted before ST, and the activation of CES prior to ST was seen in 15 out of 20 subjects. The compression force across the pelvis appeared to reduce the onset delay of GM, but it had no such effect on ES in asymptomatic subjects [27].

Another 9 studies among healthy subjects were of weak rating quality. The first study revealed a variability in the relative onset times among the 4 muscles (GM, HAM, IES, CES) both within and between subjects; 65 different muscle firing orders were observed [16]. However, in the second study, mean onset times of the muscles in the entire sample was calculated to determine an 'average' group activation order of IES-CES-ST-GM [17]. Electrical activities in the ST muscle appeared almost simultaneously with the activation of ES muscles [17].

The third study indicated that GM was significantly delayed $(p<0.05)$ compared with all other muscles [13]. The remaining muscles activation times were not statistically significantly different from each other [13]. In the fourth study, the consistency of activation orders within subjects (activation orders used for each set of 5 repetitions) and between subjects (activation orders used in the entire sample of 300 repetitions) was poor [23]. The results showed 6 different activation patterns used for $81.3 \%$ of the time (95\% Cl); GM activated last, with no clear activation order evident for HAM, IES, and CES [23] (Table 1).

The activation patterns in the fifth study were similar for hip extension associated with knee flexion, knee extension, 


\begin{tabular}{|c|c|c|c|c|c|c|}
\hline 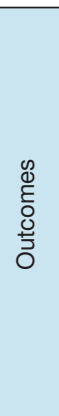 & 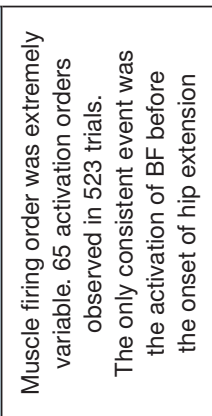 & 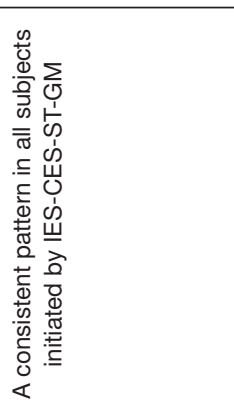 & 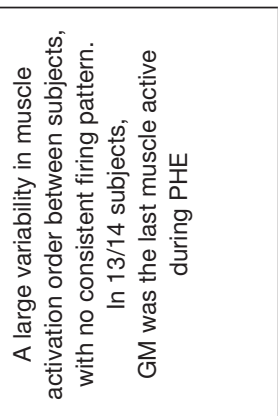 & 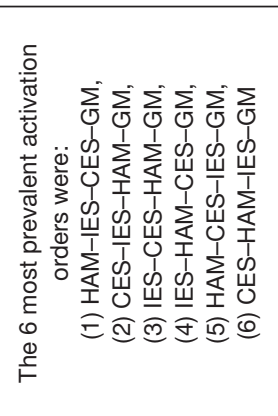 & 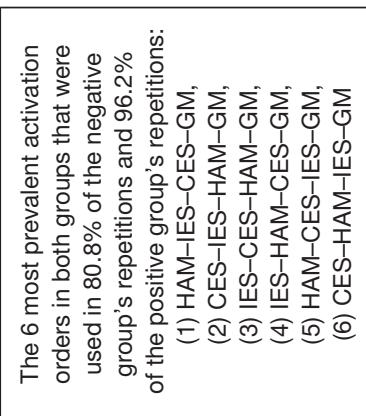 & 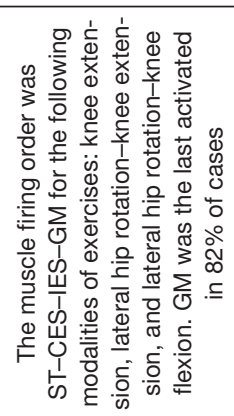 \\
\hline 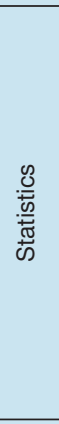 & 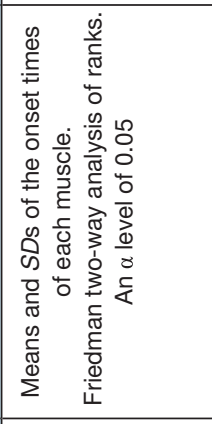 & 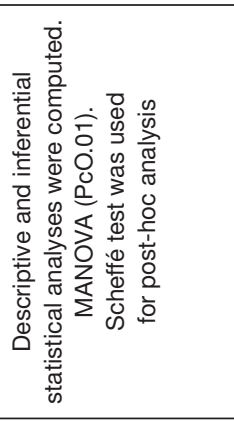 & 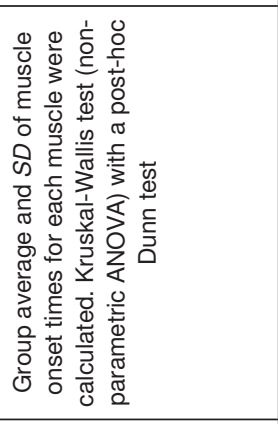 & 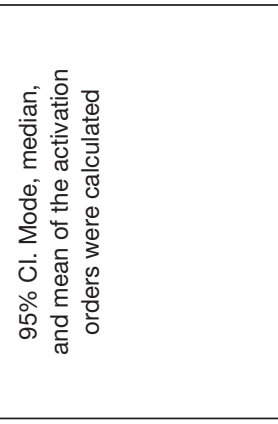 & 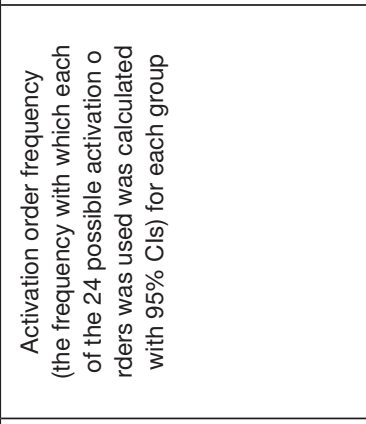 & 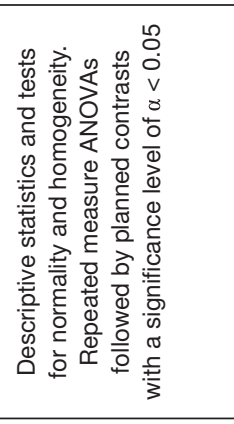 \\
\hline 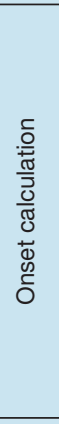 & 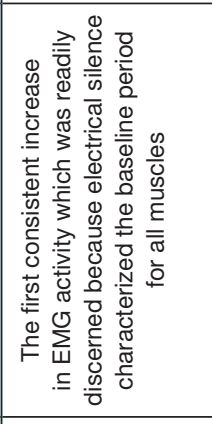 & 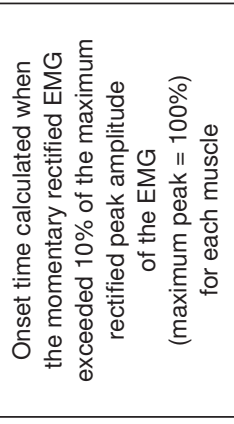 & 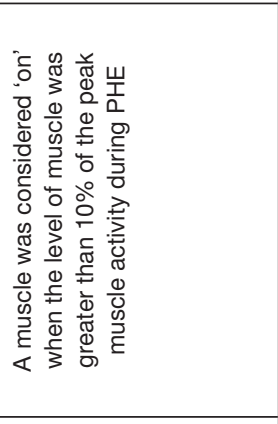 & 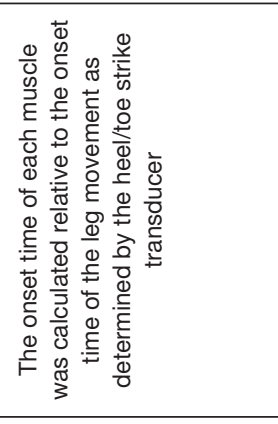 & 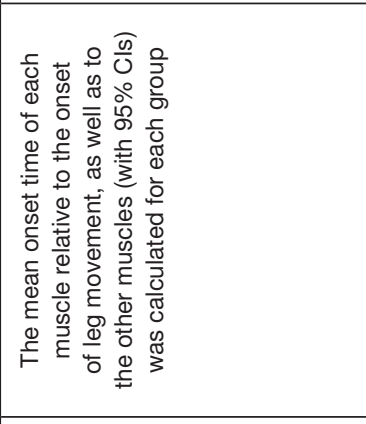 & 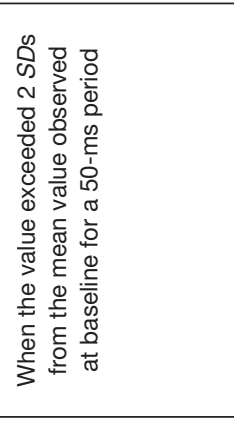 \\
\hline 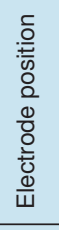 & 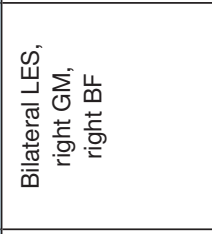 & 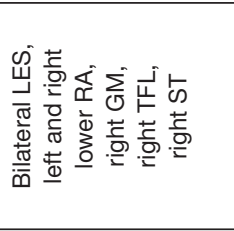 & 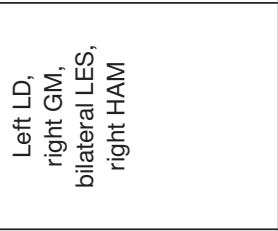 & 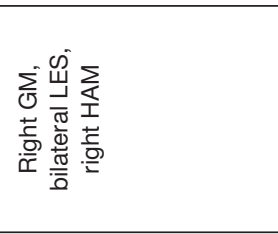 & 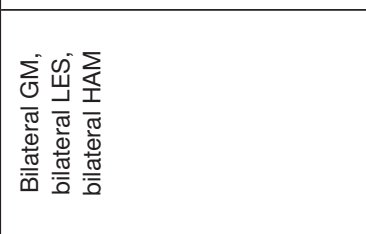 & 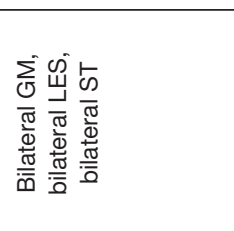 \\
\hline 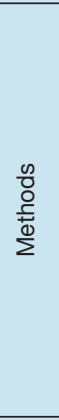 & 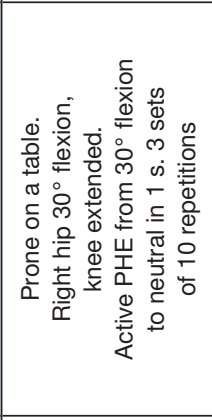 & 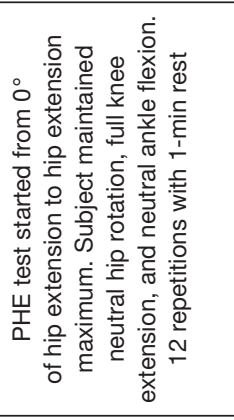 & 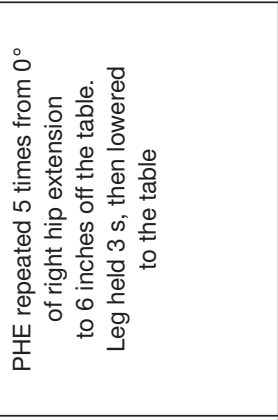 & 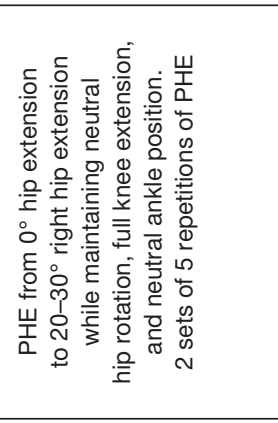 & 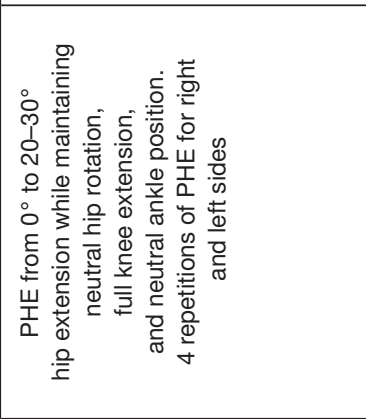 & 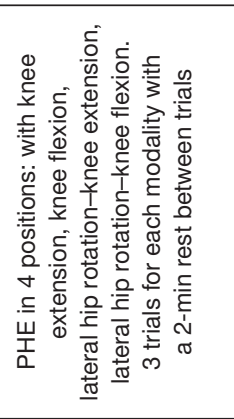 \\
\hline & 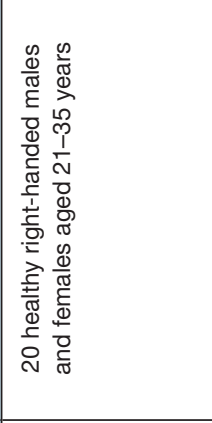 & 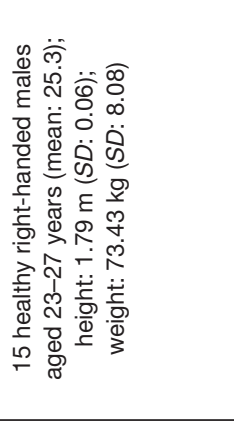 & 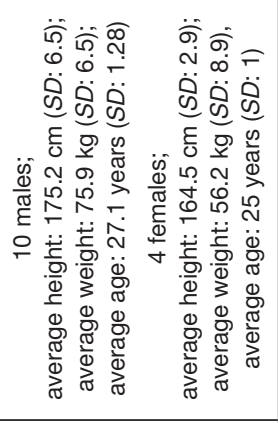 & 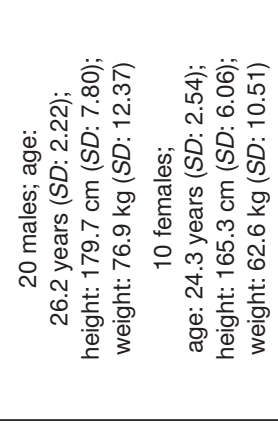 & 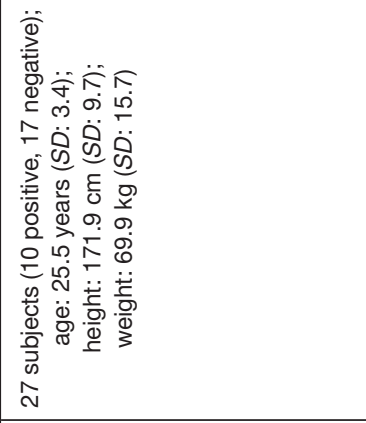 & 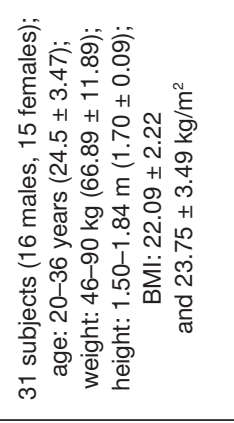 \\
\hline 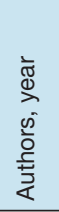 & 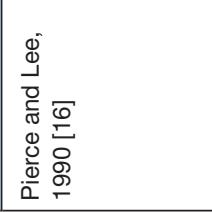 & 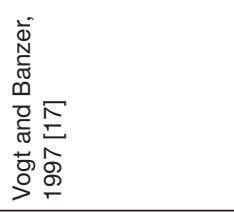 & 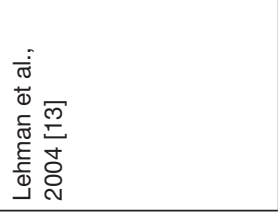 & 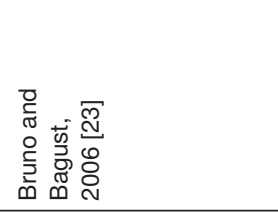 & 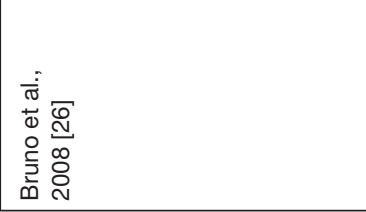 & 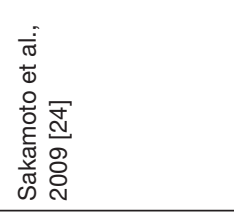 \\
\hline
\end{tabular}




\begin{tabular}{|c|c|c|c|c|}
\hline 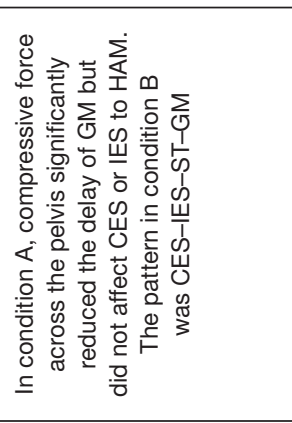 & 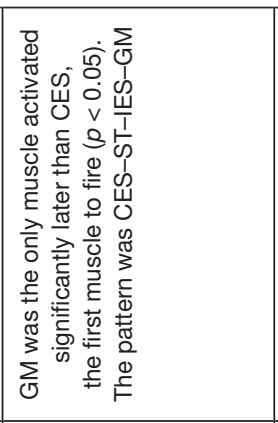 & 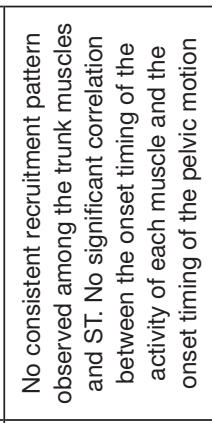 & 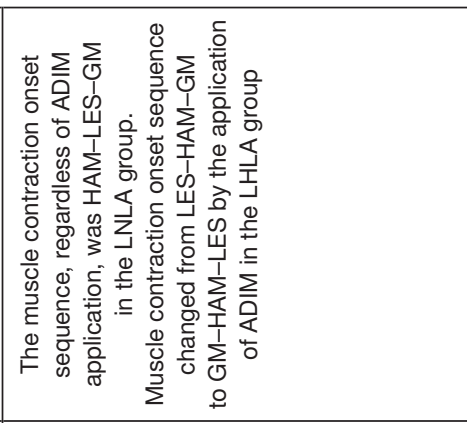 & 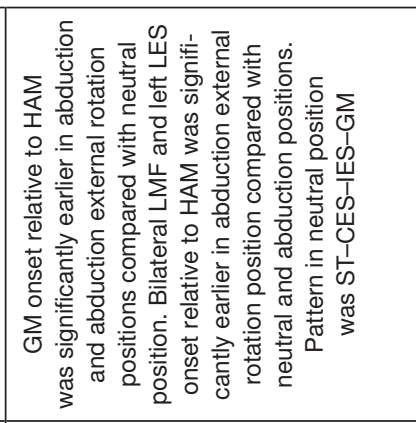 \\
\hline & 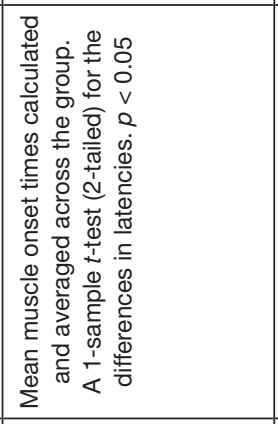 & 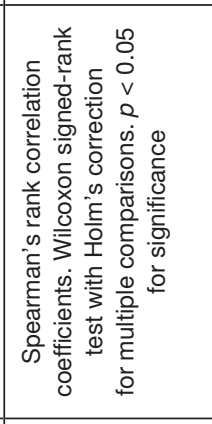 & 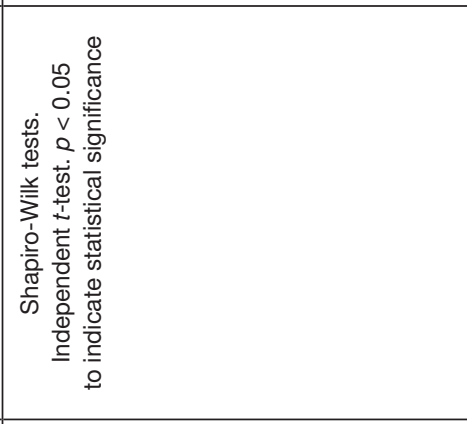 & 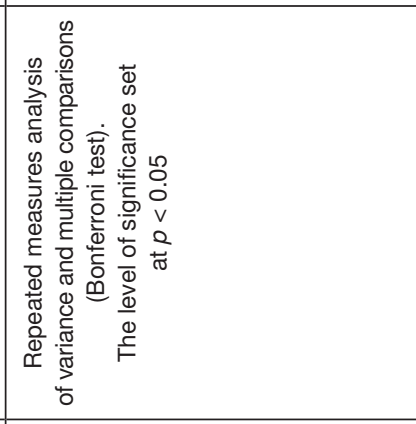 \\
\hline 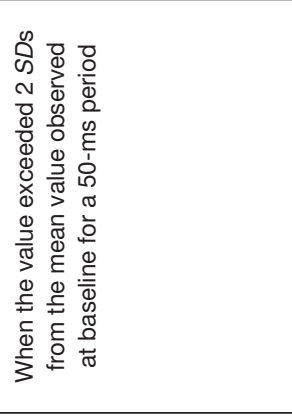 & 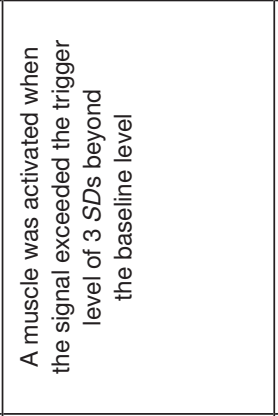 & 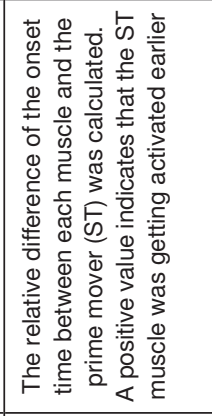 & 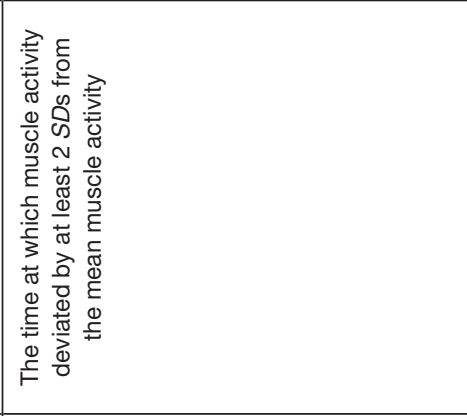 & 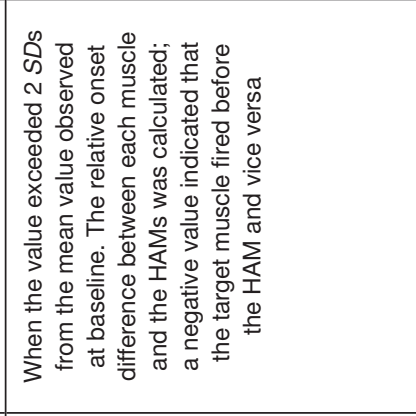 \\
\hline 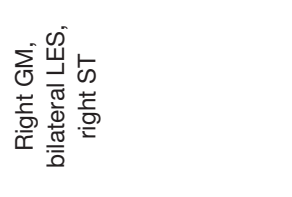 & 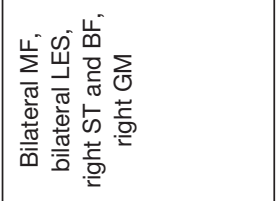 & 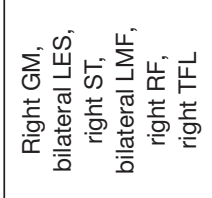 & 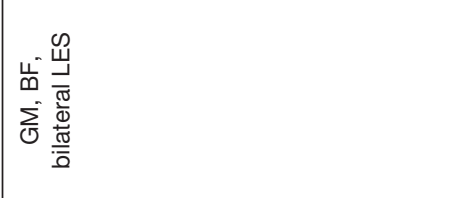 & 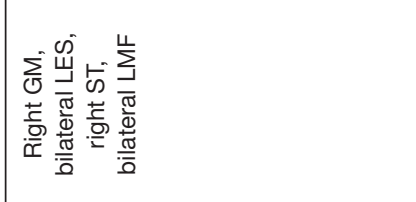 \\
\hline 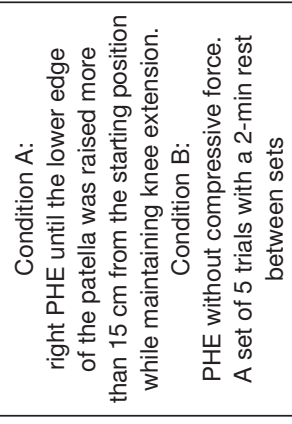 & 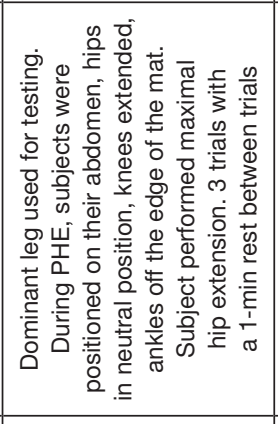 & 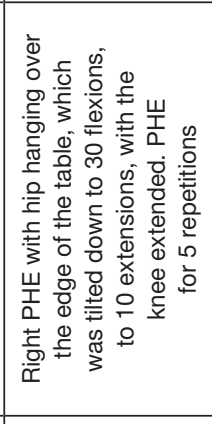 & 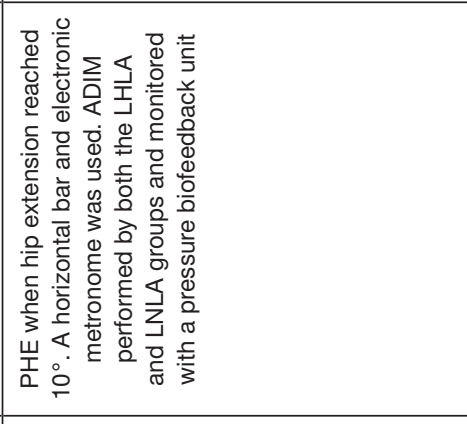 & 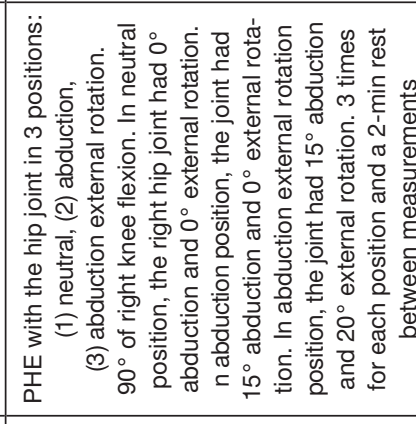 \\
\hline 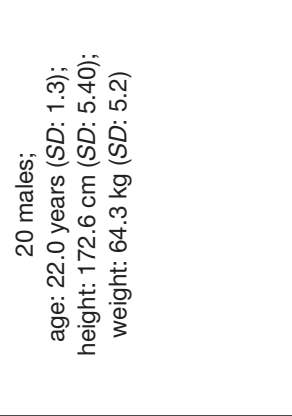 & 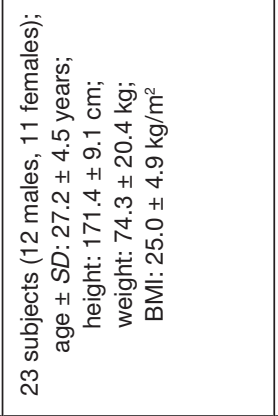 & 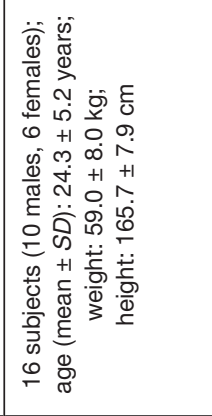 & 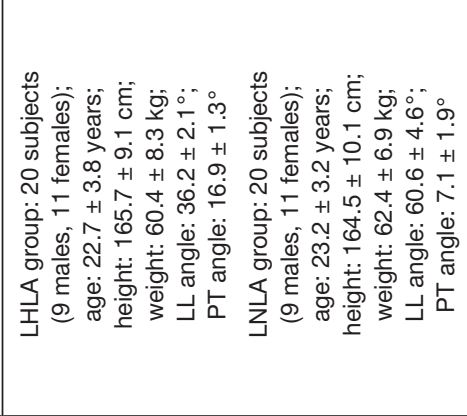 & 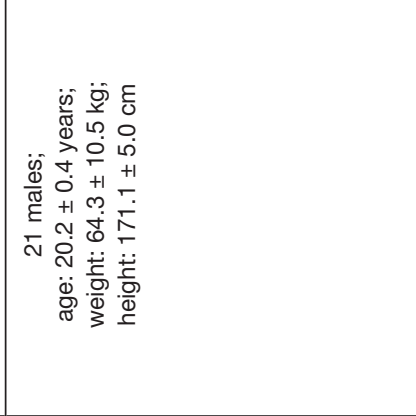 \\
\hline 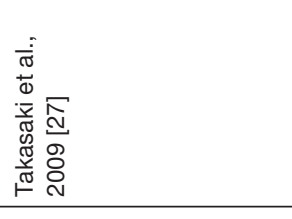 & 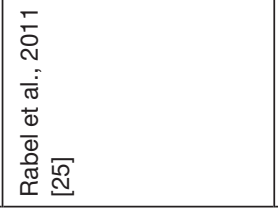 & 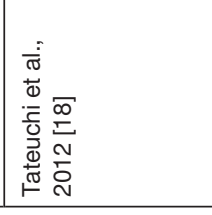 & 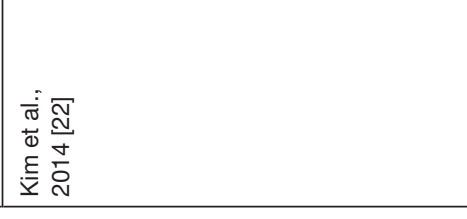 & 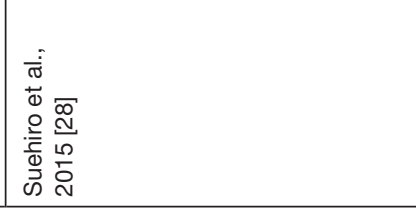 \\
\hline
\end{tabular}




\begin{tabular}{|c|c|c|c|c|}
\hline 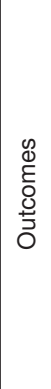 & 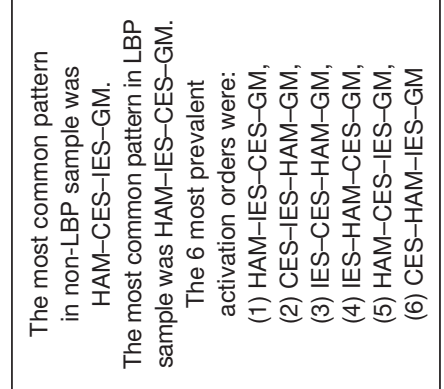 & 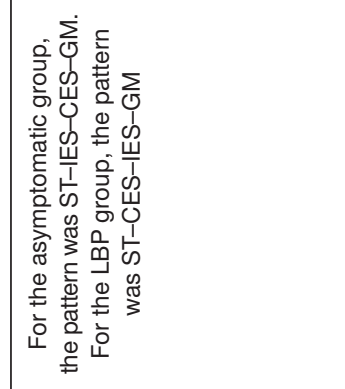 & 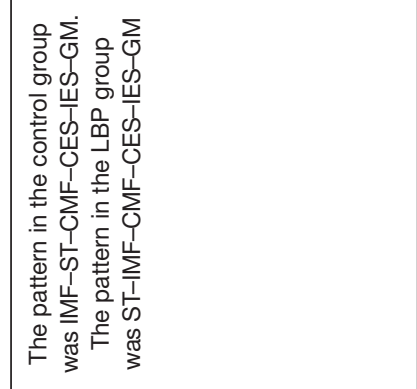 & 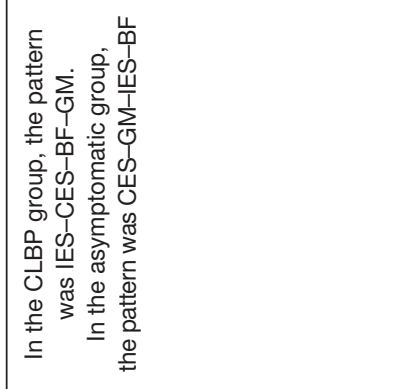 \\
\hline & 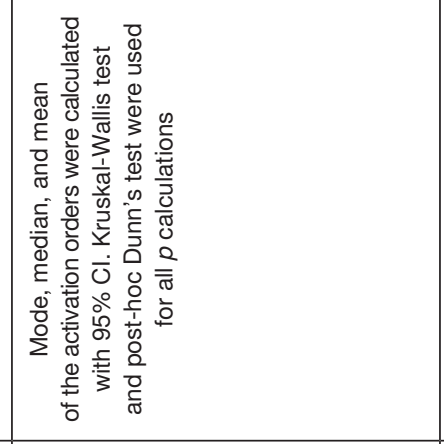 & 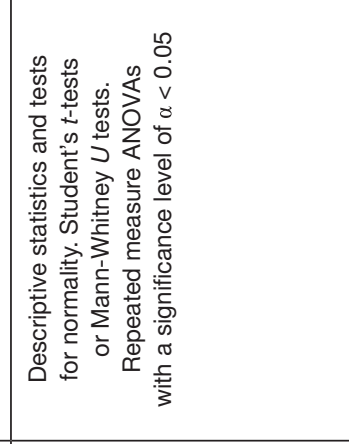 & 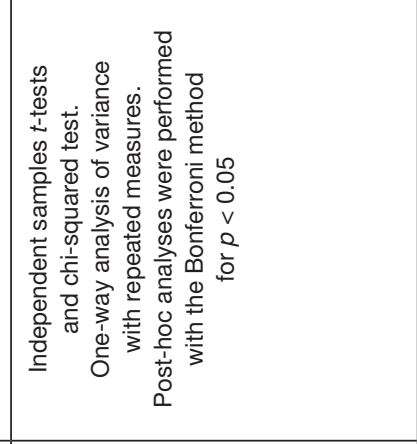 & 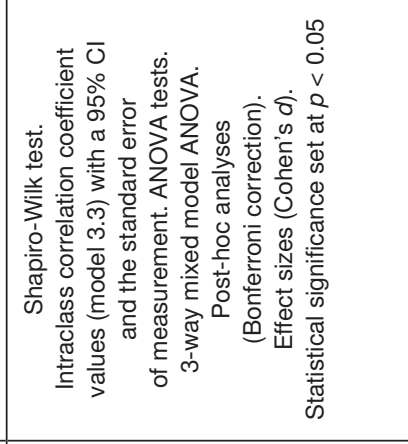 \\
\hline & 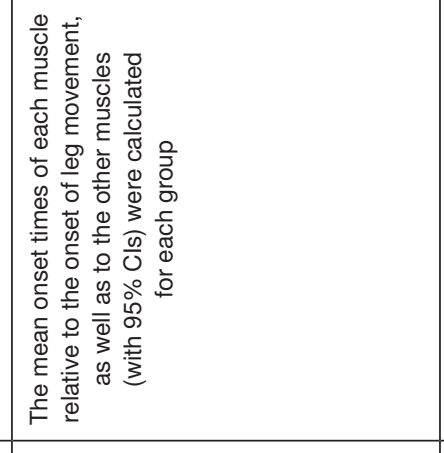 & 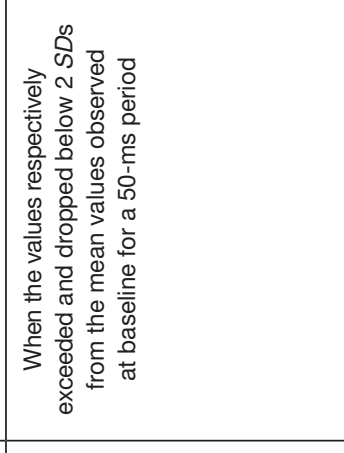 & 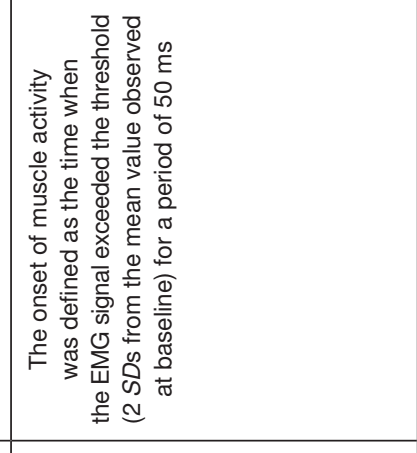 & 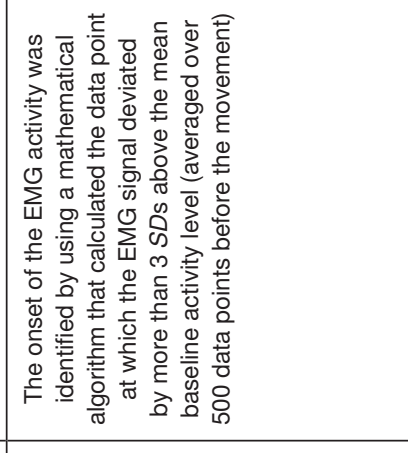 \\
\hline & 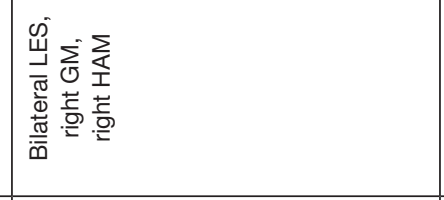 & 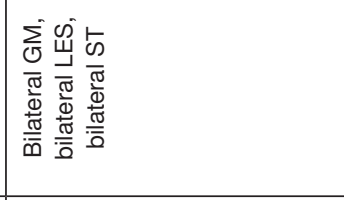 & 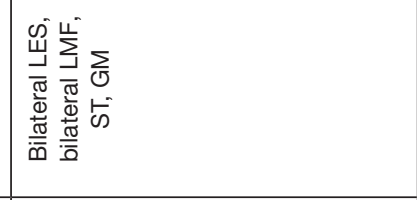 & 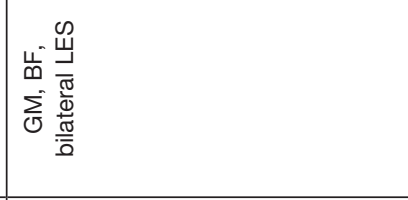 \\
\hline 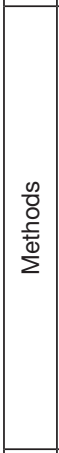 & 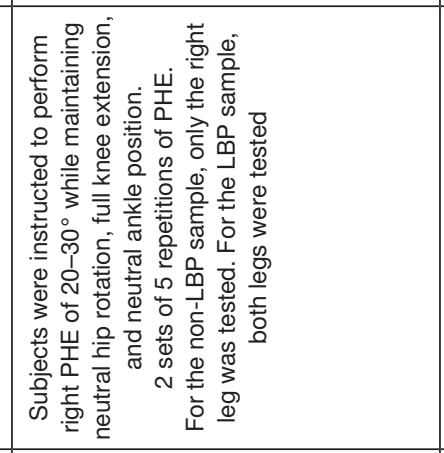 & 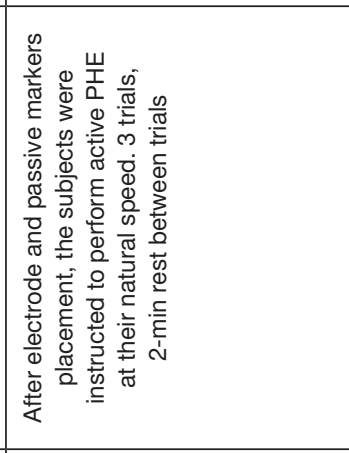 & 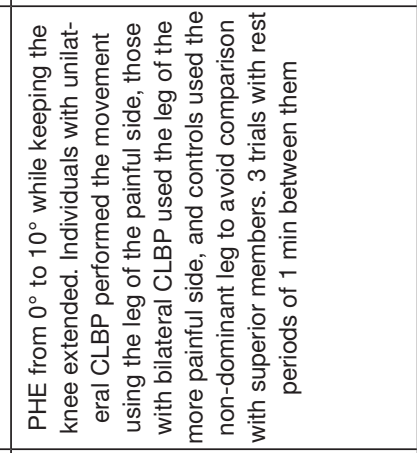 & 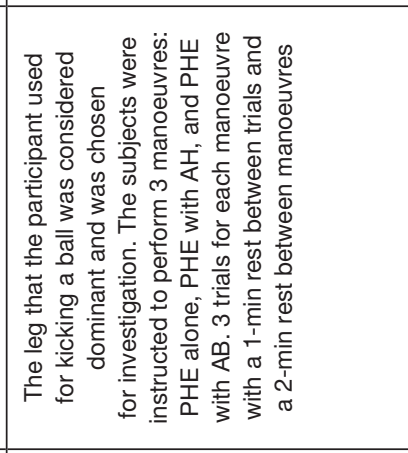 \\
\hline & 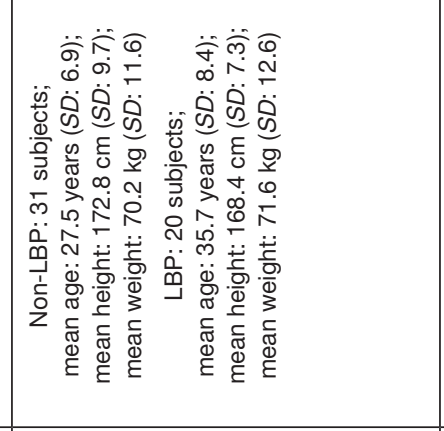 & 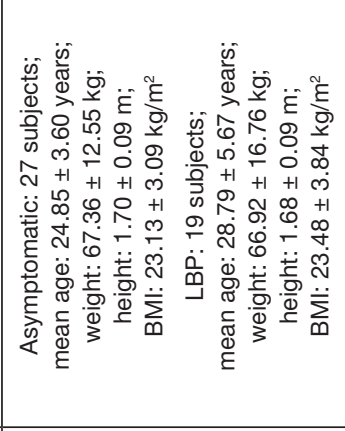 & 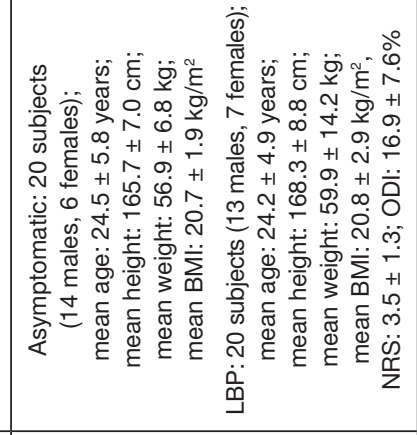 & 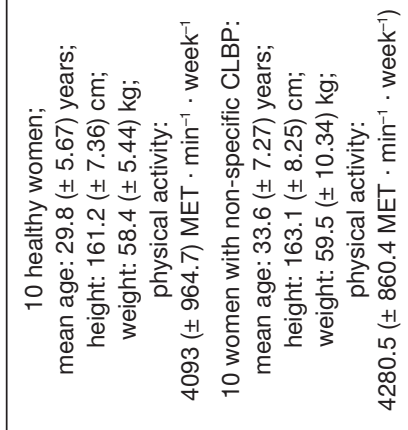 \\
\hline & 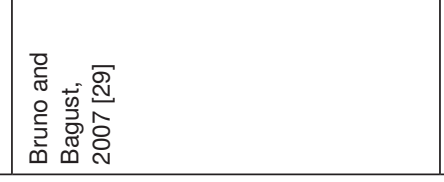 & 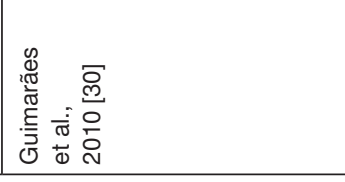 & 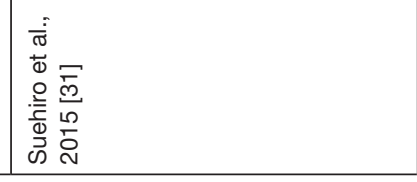 & 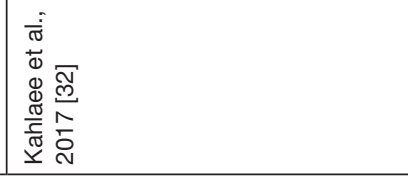 \\
\hline
\end{tabular}


Table 3. Results of quality assessment

\begin{tabular}{|c|c|c|c|c|c|c|c|c|c|}
\hline Authors, year & $\begin{array}{l}(1) \\
\text { Selection } \\
\text { bias }\end{array}$ & $\begin{array}{c}(2) \\
\text { Study } \\
\text { design }\end{array}$ & $\begin{array}{c}\text { (3) } \\
\text { Confounders }\end{array}$ & $\begin{array}{c}(4) \\
\text { Blinding }\end{array}$ & $\begin{array}{c}\text { (5) } \\
\text { Data } \\
\text { collection } \\
\text { methods }\end{array}$ & $\begin{array}{c}\text { (6) } \\
\text { Sample } \\
\text { size }\end{array}$ & $\begin{array}{c}\text { (7) } \\
\text { Withdrawals } \\
\text { and drop-outs }\end{array}$ & $\begin{array}{c}\text { (8) } \\
\text { Analyses }\end{array}$ & $\begin{array}{l}\text { Global } \\
\text { rating }\end{array}$ \\
\hline Pierce and Lee, 1990 [16] & 2 & 2 & 3 & 3 & 1 & 2 & 1 & 2 & Weak \\
\hline Vogt and Banzer, 1997 [17] & 3 & 2 & 3 & 3 & 1 & 3 & 1 & 3 & Weak \\
\hline Lehman et al., 2004 [13] & 2 & 2 & 3 & 3 & 3 & 3 & 1 & 3 & Weak \\
\hline Bruno and Bagust, 2006 [23] & 2 & 2 & 3 & 3 & 3 & 2 & 1 & 2 & Weak \\
\hline Bruno and Bagust, 2007 [29] & 2 & 2 & 3 & 3 & 3 & 2 & 1 & 1 & Weak \\
\hline Bruno et al., 2008 [26] & 2 & 2 & 2 & 3 & 3 & 2 & 1 & 1 & Moderate \\
\hline Sakamoto et al., 2009 [24] & 3 & 2 & 3 & 3 & 3 & 2 & 1 & 2 & Weak \\
\hline Takasaki et al., 2009 [27] & 2 & 2 & 2 & 3 & 2 & 2 & 1 & 3 & Moderate \\
\hline Guimarães et al., 2010 [30] & 3 & 2 & 1 & 3 & 3 & 2 & 1 & 1 & Weak \\
\hline Rabel et al., 2011 [25] & 2 & 2 & 3 & 3 & 2 & 2 & 1 & 2 & Weak \\
\hline Tateuchi et al., 2012 [18] & 3 & 2 & 3 & 2 & 3 & 3 & 1 & 2 & Weak \\
\hline Kim and Kim, 2014 [22] & 3 & 2 & 2 & 3 & 3 & 2 & 1 & 2 & Weak \\
\hline Suehiro et al., 2015 [28] & 3 & 2 & 1 & 3 & 3 & 2 & 1 & 3 & Weak \\
\hline Suehiro et al., 2015 [31] & 2 & 2 & 1 & 3 & 3 & 1 & 1 & 2 & Moderate \\
\hline Kahlaee et al., 2017 [32] & 3 & 2 & 1 & 3 & 1 & 3 & 1 & 1 & Weak \\
\hline
\end{tabular}

1 - strong, 2 - moderate, 3 - weak

and lateral rotation and knee flexion, starting with ST, followed by ES, and then GM $(p<0.0001)$ [24]. The sixth study examined the difference in intermuscular timing between the firing of the first muscle and all remaining muscles during the PHE test. It was found that GM was the only muscle activated significantly later than CES (the first muscle to fire). Also, there were no significant differences in the latency between the CES, IES, and HAM muscles during $\operatorname{PHE}(p=0.004)$ [25].

Relations between balance of hip and trunk muscle temporal patterns, pelvic motion, and low back muscle activity during PHE were examined in the seventh study [18]. The results implied that there was no consistent recruitment pattern among trunk muscles and ST. The mean onset time of all hip and trunk muscles except GM was earlier than the onset of leg movement. The onset timing of GM was delayed significantly relative to the onset timing of contralateral multifidus (CMF) ( $p=0.027)$, ipsilateral multifidus (IMF) $(p=0.008)$, CES $(p=0.038)$, IES $(p=0.035)$, and ST $(p=0.009)$. The delay of the hip and trunk muscles relative to ST firing was significantly associated with an increase of the anterior pelvic tilt during hip extension [18].

The authors of the eighth study investigated the effect of the abdominal drawing-in manoeuvre (ADIM) on muscle onset time in HAM, GM, and ES during PHE in subjects with hyper-lordotic angle but without LBP [22]. The subjects were divided into 2 groups: with lumbar hyper-lordotic angle (LHLA) and with lumbar normal lordotic angle (LNLA). No significant differences were observed between the groups in the differential values of HAM or ES onset time with or without ADIM application $(p>0.05)$. Only GM in the LHLA group presented a significant difference during $\operatorname{ADIM}(p<0.01)$ [22].

The results revealed that the muscle contraction onset sequence, regardless of ADIM, was HAM-ES-GM in the LNLA group. However, the muscle contraction onset sequence changed from ES-HAM-GM to GM-HAM-ES with the application of ADIM in the LHLA group [22].

Finally, the ninth study investigated the effect of 3 hip positions - neutral, abduction, and abduction with external rotation - on muscle onset time during PHE with knee flexion [28]. GM was the last muscle to activate in the neutral position. However, GM onset relative to HAM was significantly earlier with hip abduction and with hip abduction with external rotation compared with that with hip in neutral position. Bilateral multifidus (MF) and CES onset relative to HAM was significantly earlier in the abduction with external rotation position than in the neutral and abduction positions. The sequence of muscle contraction was HAM-CES-IES-GM in the neutral position, IES-GM-HAM-CES in the abduction position, and CES-IES-GM-HAM in the abduction with external rotation position [28].

\section{Differences in activation patterns between asymptomatic and LBP subjects}

One of the 4 studies in LBP subjects was of moderate rating quality [31]. It revealed that the onset of GM was delayed significantly relative to the onset of IMF $(p<0.001)$, $\operatorname{CMF}(p<0.001)$, IES $(p<0.01)$, and CES $(p<0.001)$ in both the control and CLBP group. There were no significant differences in the onset times of GM $(p=0.32)$ or IES $(p=0.11)$ between the groups. However, the onset times of bilateral MF $(p<0.001)$ and CES $(p=0.001)$ were delayed in the CLBP group compared with the control group [31].

The second study demonstrated that the onset of GM was significantly delayed in both the symptomatic $(95 \% \mathrm{Cl}$; $p<0.001)$ and the asymptomatic leg $(95 \% \mathrm{Cl} ; p<0.001)$ in the LBP group compared with the non-LBP group [29]. CES onset was also significantly delayed in the symptomatic leg in the LBP group $(95 \% \mathrm{Cl} ; p<0.05)$, but no significant differ- 
ence was found with the asymptomatic leg. There was a high degree of variability in the activation patterns. Six activation orders were the most prevalent in both samples, with none showing a strong predominance [29] (Table 2).

The most common muscle to become active first was HAM in all the 3 groups. The most common muscle to become active second was CES in the non-LBP sample and IES in both legs in the LBP sample. The most common muscle to become active third was IES in the non-LBP sample and CES in both legs in the LBP sample. The most common muscle to become active fourth was GM in all 3 groups [29].

The authors of the third study suggested that the patterns in the asymptomatic group were initiated by ST, followed by IES, CES, and finished by GM. The patterns in the LBP group were initiated by ST, followed by CES, IES, and finally GM [30]. ANOVAs demonstrated significant differences in latencies for $\mathrm{GM}$ in relation to other muscles in both groups (non-LBP: $F>41.78$; LBP: $F>23.64 ; p<0.001$ ). No significant differences were found between groups regarding latencies for any investigated muscle [30].

Finally, the effect of abdominal hollowing $(\mathrm{AH})$ and abdominal bracing $(A B)$ manoeuvres on the activity pattern of lumbopelvic muscles during PHE in participants with or without non-specific CLBP was investigated [32]. There were no significant differences in muscle activation onset times between patients and asymptomatic participants in any of the manoeuvres (PHE, PHE $+\mathrm{AH}, \mathrm{PHE}+\mathrm{AB})$, except for CES in PHE $(p=0.03)$, which was delayed in the CLBP group compared with the asymptomatic group [32].

\section{Discussion}

Concerning the patterns in healthy individuals, there was moderate evidence [26, 27] and weak evidence [13, 17, 24, $25]$ that the HAM and ES muscles were almost simultaneously activated before the onset of the leg movement during $\mathrm{PHE}$, while they exhibited inconsistent and variable patterns in the remaining studies [16, 18, 22, 23, 28]. GM was significantly delayed and constituted the last muscle to activate during PHE in all studies which examined healthy participants.

Regarding the activation patterns in CLBP subjects, there was moderate evidence [31] and weak evidence [29, 32] that the onset of CES was significantly delayed when compared with non-LBP participants, while no significant differences were found for the other muscles (HAM, IES, GM). GM was significantly delayed relative to the other muscles in both CLBP and control group.

Only one study revealed that the onset of GM activity was delayed in individuals with LBP relative to the control group during PHE [29]. This inconsistency may originate from the difference in the proportion of individuals with LBP who presented abnormal lumbar spine motion during PHE between other studies and the report of this study [29]. In this regard, a subsequent report suggested that the delay in the onset of GM activity during PHE was associated with abnormal motion, such as rotation, lateral flexion, and extension, of the lumbar spine in individuals with or without LBP $[22,26]$.

The results of this systematic review support the conclusions drawn in previous studies [33, 34] that proximal musculature was activated first in order to create stability and a feedforward mechanism needed to move the limb. This finding suggests that healthy individuals can recruit the stabilizing musculature (ES) in anticipation of lower extremity movement associated with PHE to achieve lumbopelvic stability. The stabilization cascade occurred very rapidly, and the only muscle that was significantly delayed, possibly owing to its primary role in producing torque on the limb, was GM [25].

Therefore, the delayed activation of CES in CLBP subjects reduced the spinal control and pelvic stability at initiation of leg motion during PHE. Inability to stabilize the lumbopelvic region effectively during limb movement may contribute to continued trauma to spinal structures, resulting in sustained or repeated episodes of LBP [35]. In this regard, it was reported that delayed MF and CES activity increased anterior pelvic tilt in healthy individuals during PHE [18]. Moreover, a later onset of the MF and ES muscles activity was observed during rapid arm movements and reaching task in patients with LBP and segmental instability relative to control subjects [36]. These results with the PHE task refute prior literature reports that GM should fire early in the sequence [37]. It was found that it was normal for GM to fire last.

The results of this systematic review implied delayed GM activation relative to the other 3 muscles in healthy and LBP subjects but failed to reach agreement on the delay magnitude. This is due to methodological differences between the studies, such as PHE position, repetitions, number of muscles evaluated, gender of participants, control of movement speed, and onset calculation.

Although most of the studies in this review used PHE test starting from neutral to maximum hyperextension, in 2 studies the participants started the test with hip in $30^{\circ}$ flexion to maximum extension $[16,28]$. These papers suggested that those subjects with a limited end range of extension would be performing trunk hyperextension instead of hip extension during prone leg lifting. This would not provide valid information on the muscle firing order during active PHE.

Concerning the number of muscles evaluated, all studies investigated GM, ES, and HAM; however, some studies investigated other muscles in addition to the mentioned above. One study examined rectus abdominus (RA) and tensor fasciae latae (TFL) [17]. Another study investigated latissimus dorsi (LD) [13], while MF muscles were considered in 2 studies [18, 28]. It was suggested that if more muscles were evaluated, it would be difficult to find a consistent pattern of muscle activation [38].

Referring to the speed of movement, PHE was performed at a low speed in 3 of the included studies [16-18] and with a high speed in 1 study [31]; the remaining studies did not specify the speed of limb movement. The studies indicated that performing movement at a low speed reduced the frequency of response of trunk muscles and increased the variability [39, 40].

Another difference was the participants' gender. Some studies investigated both genders and found variable patterns $[13,16,23]$. Others investigated only males and reported consistency [17], although 1 study implied a pattern variability in male participants [26]. One study included only females to compare the pattern in healthy and CLBP subjects and no difference was found in the pattern except for CES, which was delayed in CLBP subjects [32]. Future electromyographic research should examine if differences in muscle firing patterns exist between males and females. It was observed that during certain movement tasks, electromyographic signal amplitude levels were consistently higher in the lumbar MF in women as compared with men [41].

As for the method of onset calculation, the studies included in this review used methods that varied from a researcher's visual assessment of the signal [16] to the application of the percentage of the maximum rectified peak amplitude $[13,17,18]$ or a certain number of standard deviations above the baseline average [22-32]. It was suggested 
that 2 standard deviations from the mean values observed at baseline for a $50-\mathrm{ms}$ period was a reliable method and helped avoid type I (when using 1 standard deviation) and type II (3 standard deviations) methodological errors [42]. In contrast, the method of 3 standard deviations beyond the level at the beginning of the movement was considered reliable in previous reports $[43,44]$.

\section{Clinical implications}

As most studies in this review were of weak quality and at a risk of bias, there is no sufficient evidence regarding an ideal activation pattern in healthy individuals that could be used as a reference point in comparisons with abnormal activation patterns in LBP subjects. Large, high-quality studies are needed to identify faulty patterns and to evaluate the movement patterns during PHE to indicate changes, such as pelvic anteversion or lumbar spine rotation, which could generate excessive stress and pain in the spine.

\section{Limitations of the review}

The first limitation is that only studies that investigated muscle onset time were included but not studies that examined the amplitude of muscle contractions. The second limitation is that the searching process was limited to the English language.

\section{Conclusions}

Despite no strong evidence, HAM and ES muscles are activated simultaneously and early before the onset of the leg movement, and GM is significantly delayed in relation to the HAM and ES muscles and to the onset of the leg movement. The CES muscles are delayed in LBP subjects in comparison with healthy individuals. GM is significantly delayed in LBP subjects who present abnormal lumbar motions when compared with healthy people.

\section{Acknowledgments}

The authors express their deep thanks, respect, and appreciation to Dr. Amir A. Beltagi, lecturer in the Department of Biomechanics, Faculty of Physical Therapy, Cairo University, for his kind help in searching databases.

\section{Disclosure statement}

No author has any financial interest or received any financial benefit from this research.

\section{Conflict of interest}

The authors state no conflict of interest.

\section{References}

1. Hoy D, Brooks P, Blyth F, Buchbinder R. The epidemiology of low back pain. Best Pract Res Clin Rheumatol. 2010;24(6):769-781; doi: 10.1016/j.berh.2010.10.002.

2. O'Sullivan P. Diagnosis and classification of chronic low back pain disorders: maladaptive movement and motor control impairments as underlying mechanism. Man Ther. 2005;10(4):242-255; doi: 10.1016/j.math.2005.07.001.

3. Scannell JP, McGill SM. Lumbar posture - should it, and can it, be modified? A study of passive tissue stiffness and lumbar position during activities of daily living. Phys Ther. 2003;83(10):907-917; doi: 10.1093/ptj/83. 10.907.
4. Smith A, O'Sullivan $P$, Straker L. Classification of sagittal thoraco-lumbo-pelvic alignment of the adolescent spine in standing and its relationship to low back pain. Spine. 2008;33(19):2101-2107; doi: 10.1097/BRS.0b013e318 17 ec3b0.

5. Hodges P, van den Hoorn W, Dawson A, Cholewicki J. Changes in the mechanical properties of the trunk in low back pain may be associated with recurrence. J Biomech. 2009;42(1):61-66; doi: 10.1016/j.jbiomech.2008.10.001.

6. Lee J-H, Hoshino Y, Nakamura K, Ooi Y. Trunk muscle imbalance as a risk factor of the incidence of low back pain: a 5-year prospective study. J Neuromusculoskelet Syst. 1999;7:97-101.

7. Page P, Frank CC, Lardner R. Assessment and treatment of muscle imbalance: the Janda approach. Champaign: Human Kinetics; 2010.

8. Arab AM, Ghamkhar L, Emami M, Nourbakhsh MR. Altered muscular activation during prone hip extension in women with and without low back pain. Chiropr Man Therap. 2011;19:18; doi: 10.1186/2045-709X-19-18.

9. McGill SM, Grenier S, Kavcic N, Cholewicki J. Coordination of muscle activity to assure stability of the lumbar spine. J Electromyogr Kinesiol. 2003;13(4):353-359; doi: 10.1016/s1050-6411(03)00043-9.

10. Janda V. On the concept of postural muscles and posture in man. Aust J Physiother. 1983;29(3):83-84; doi: 10.1016/S0004-9514(14)60665-6.

11. Sahrmann SA. Posture and muscle imbalance: faulty lumbar-pelvic alignment and associated musculoskeletal pain syndromes. Orthop Div Rev. 1992;13-20.

12. Leinonen V, Kankaanpää M, Airaksinen $O$, Hänninen $O$. Back and hip extensor activities during trunk flexion/ extension: effects of low back pain and rehabilitation. Arch Phys Med Rehabil. 2000;81(1):32-37; doi: 10.1016/ s0003-9993(00)90218-1.

13. Lehman GJ, Lennon D, Tresidder B, Rayfield B, Poschar M. Muscle recruitment patterns during the prone leg extension. BMC Musculoskelet Disord. 2004;5:3; doi: 10.1186/1471-2474-5-3.

14. Gregory DE, Callaghan JP. Prolonged standing as a precursor for the development of low back discomfort: an investigation of possible mechanisms. Gait Posture. 2008;28(1):86-92; doi: 10.1016/j.gaitpost.2007.10.005.

15. Dunk NM, Callaghan JP. Lumbar spine movement patterns during prolonged sitting differentiate low back pain developers from matched asymptomatic controls. Work. 2010;35(1):3-14; doi: 10.3233/WOR-2010-0953.

16. Pierce $M N$, Lee WA. Muscle firing order during active prone hip extension. J Orthop Sports Phys Ther. 1990; 12(1):2-9; doi: 10.2519/jospt.1990.12.1.2.

17. Vogt L, Banzer W. Dynamic testing of the motor stereotype in prone hip extension from neutral position. Clin Biomech. 1997;12(2):122-127; doi: 10.1016/s0268-0033 (96)00055-1.

18. Tateuchi H, Taniguchi M, Mori N, Ichihashi N. Balance of hip and trunk muscle activity is associated with increased anterior pelvic tilt during prone hip extension. J Electromyogr Kinesiol. 2012;22(3):391-397; doi: 10.1016/j.jelekin.2012.03.003.

19. Jarosz BS. Muscle activation patterns during the prone hip extension test: a review of the literature. Chiropr $\mathrm{J}$ Aust. 2010;40(3):103-108.

20. Moher D, Liberati A, Tetzlaff J, Altman DG, PRISMA Group. Preferred reporting items for systematic reviews and meta-analyses: the PRISMA statement. 
PLoS Med. 2009;6(7):e1000097; doi: 10.1371/journal. pmed.1000097.

21. Thomas BH, Ciliska D, Dobbins M, Micucci S. A process for systematically reviewing the literature: providing the research evidence for public health nursing interventions. Worldviews Evid Based Nurs. 2004;1(3): 176-184; doi: 10.1111/j.1524-475X.2004.04006.x.

22. Kim T, Woo Y, Kim Y. Effect of abdominal drawing-in maneuver during hip extension on the muscle onset time of gluteus maximus, hamstring, and lumbar erector spinae in subjects with hyperlordotic lumbar angle. J Physiol Anthropol. 2014;33(1):34; doi: 10.1186/18806805-33-34.

23. Bruno PA, Bagust J. An investigation into the withinsubject and between-subject consistency of motor patterns used during prone hip extension in subjects without low back pain. Clin Chiropr. 2006;9(1):11-20; doi: 10.1016/j.clch.2006.01.003.

24. Sakamoto ACL, Teixeira-Salmela LF, de Paula-Goulart FR, de Morais Faria CDC, Guimarães CQ. Muscular activation patterns during active prone hip extension exercises. J Electromyogr Kinesiol. 2009;19(1):105-112; doi: 10.1016/j.jelekin.2007.07.004.

25. Rabel MC, Rosenthal F, Smith J, Bennett H. Hip and trunk muscle timing during selected movement tasks: an electromyographic analysis. Int J Sci Inform. 2011; 1(1):1-8.

26. Bruno PA, Bagust J, Cook J, Osborne N. An investigation into the activation patterns of back and hip muscles during prone hip extension in non-low back pain subjects: normal vs. abnormal lumbar spine motion patterns. Clin Chiropr. 2008;11(1):4-14; doi: 10.1016/j.clch.2008. 01.001.

27. Takasaki H, lizawa T, Hall T, Nakamura T, Kaneko S. The influence of increasing sacroiliac joint force closure on the hip and lumbar spine extensor muscle firing pattern. Man Ther. 2009;14(5):484-489; doi: 10.1016/j.math. 2008.11.003.

28. Suehiro T, Mizutani M, Ishida H, Kobara K, Fujita D, Osaka $\mathrm{H}$, et al. Effect of abduction and external rotation of the hip joint on muscle onset time during prone hip extension with knee flexion. J Phys Ther Sci. 2015; 27(1):289-291; doi: 10.1589/jpts.27.289.

29. Bruno PA, Bagust J. An investigation into motor pattern differences used during prone hip extension between subjects with and without low back pain. Clin Chiropr. 2007;10(2):68-80; doi: 10.1016/j.clch.2006.10.002.

30. Guimarães CQ, Sakamoto ACL, Laurentino GEC, Teixeira-Salmela LF. Electromyographic activity during active prone hip extension did not discriminate individuals with and without low back pain. Rev Bras Fizioter. 2010; 14(4):351-357; doi:10.1590/s1413-35552010005000017.

31. Suehiro T, Mizutani M, Ishida $H$, Kobara K, Osaka H, Watanabe S. Individuals with chronic low back pain demonstrate delayed onset of the back muscle activity during prone hip extension. J Electromyogr Kinesiol. 2015;25(4): 675-680; doi: 10.1016/j.jelekin.2015.04.013.

32. Kahlaee AH, Ghamkhar L, Arab AM. Effect of the abdominal hollowing and bracing maneuvers on activity pattern of the lumbopelvic muscles during prone hip extension in subjects with or without chronic low back pain: a preliminary study. J Manipulative Physiol Ther. 2017;40(2):106-117; doi: 10.1016/j.jmpt.2016.10.009.

33. Aruin AS, Latash ML. Directional specificity of postural muscles in feed-forward postural reactions during fast voluntary arm movements. Exp Brain Res. 1995;103(2): 323-332; doi: 10.1007/BF00231718.

34. Hodges PW, Richardson CA. Contraction of the abdominal muscles associated with movement of the lower limb. Phys Ther. 1997;77(2):132-142; doi: 10.1093/ptj/ 77.2.132.

35. Richardson C, Hodges P, Hides J. Therapeutic exercise for lumbopelvic stabilization. Edinburgh: Churchill Livingstone; 2004.

36. Silfies SP, Mehta R, Smith SS, Karduna AR. Differences in feedforward trunk muscle activity in subgroups of patients with mechanical low back pain. Arch Phys Med Rehabil. 2009;90(7):1159-1169; doi: 10.1016/j.apmr. 2008.10.033.

37. Greenman PE. Principles of manual medicine, $3^{\text {rd }}$ ed. Philadelphia: Lippincott Williams \& Wilkins; 2003.

38. Lewis CL, Sahrmann SA. Muscle activation and movement patterns during prone hip extension exercise in women. J Athl Train. 2009;44(3):238-248; doi: 10.4085/ 1062-6050-44.3.238.

39. Hodges PW, Richardson CA. Relationship between limb movement speed and associated contraction of the trunk muscles. Ergonomics. 1997;40(11):1220-1230; doi: 10.1080/001401397187469.

40. Hodges PW, Richardson CA. Altered trunk muscle recruitment in people with low back pain with upper limb movement at different speeds. Arch Phys Med Rehabil. 1999;80(9):1005-1012; doi: 10.1016/s0003-9993(99) 90052-7.

41. Arokoski JP, Valta T, Airaksinen O, Kankaanpää M. Back and abdominal muscle function during stabilization exercises. Arch Phys Med Rehabil. 2001;82(8):1089-1098; doi: 10.1053/apmr.2001.23819.

42. Hodges PW, Bui BH. A comparison of computer-based methods for the determination of onset of muscle contraction using electromyography. Electroencephalogr Clin Neurophysiol. 1996;101(6):511-519; doi: 10.1016/ S0921-884X(96)95190-5.

43. Di Fabio RP. Reliability of computerized surface electromyography for determining the onset of muscle activity. Phys Ther. 1987;67(1):43-48; doi: 10.1093/ptj/67.1.43.

44. Bolgla LA, Malone TR, Umberger BR, UhI TL. Reliability of electromyographic methods used for assessing hip and knee neuromuscular activity in females diagnosed with patellofemoral pain syndrome. J Electromyogr Kinesiol. 2010;20(1):142-147; doi: 10.1016/j.jelekin.2008. 11.008 . 